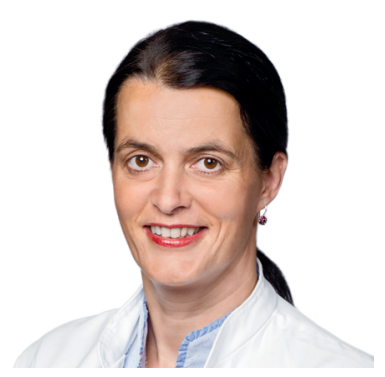

\title{
Assoziationen statt Kausalitäten: zur Empfehlung von Vitamin-D-Einnahme gegen Allergien
}

\author{
Claudia Traidl-Hoffmann ${ }^{\mathrm{a}, \mathrm{b}}$
}

aLehrstuhl und Institut für Umweltmedizin, UNIKA-T, Technische Universität München und Helmholtzzentrum München

${ }^{b}$ Ambulanz für Umweltmedizin, Klinikum Augsburg

Abstractübersetzung aus Savilahti EM, Mäkitie O, Kukkonen AK, Andersson S, Viljakainen H, Savilahti E, Kuitunen M: Serum 25-hydroxyvitamin d in early childhood is nonlinearly associated with allergy. Int Arch Allergy Immunol 2016;170:141-148.

Serumspiegel von 25-Hydroxyvitamin D im frühen Kindesalter sind nichtlinear mit Allergie assoziiert

\section{Schlüsselwörter}

25-Hydroxyvitamin D · Allergie · Probiotika · Sensibilisierung · Nabelschnurblut

\section{Zusammenfassung}

Hintergrund: Vitamin D hat verschiedene immunologische Funktionen. Die Daten zur Beziehung zwischen Vitamin-D-Status und Allergien sind umstritten.

Methoden: Wir untersuchten den Zusammenhang zwischen Serumkonzentrationen von 25-Hydroxyvitamin D (25-OHD) und Allergien im Kindesalter. Die Studienpopulation $(n=819)$ war Teil einer randomisierten, doppelblinden, Placebo-kontrollierten Studie, in der Mütter, bei deren Kindern ein hohes Allergierisiko bestand, in den letzten 4 Wochen der Schwangerschaft eine Probiotika-Mischung (oder Placebo) einnahmen und ihr Kind dies nach der Geburt 6 Monate lang erhielt. Die Probanden wurden 5 Jahre lang im Hinblick auf das Auftreten von Sensibilisierung und allergischen Symptomen beobachtet und im Alter von 3 und 6 Monaten, 2 und 5 Jahren sowie im Falle allergischer Symptome medizinisch untersucht. Die 25-OHD-Spiegel wurden in Proben von Nabelschnurblut (NSB) ( $n=724)$ und in Serumproben, die im Alter von 2 Jahren genommen wurden, $(n=369)$ gemessen. Die Daten wurden in Terzile (T1-T3) und Quartile (Q1-Q4) kategorisiert. Die Beziehung zwischen 25-OHD-Spiegeln und Sensibilisierung bzw. Allergie wurde mit einer multivablen logistischen Regressionsanalyse ausgewertet.

Ergebnisse: 25-OHD-Spiegel in T2 im NSB waren mit einem höheren Risiko einer Sensibilisierung im Alter von 2 Jahren und allergischen Erkrankungen im Alter von 5 Jahren verbunden. Bei den im Alter von 2 Jahren genommenen Serumproben waren 25-OHD-Spiegel in Q3 mit einem höheren Sensibilisierungsrisiko und IgE-vermittelten Allergien im Alter von 5 Jahren verbunden. Schlussfolgerung: Die 25-OHD-Spiegel im frühen Kindesalter sind mit dem Auftreten von Allergien assoziiert, jedoch scheint der Zusammenhang nichtlinear zu sein.

C 2017 S. Karger GmbH, Freiburg

\section{KARGER}

Fax +497614520714 information@karger.com www.karger.com 


\section{Transfer in die Praxis}

\section{Hintergrund}

Um das Vitamin D hat sich ein wahrer Hype entwickelt. Es scheint gut zu sein für vieles, z.B. soll es vor chronischen Infektionen [1], Depressionen [2], vor Krebs [3] und Diabetes mellitus Typ 2 [4] schützen, und diese Liste könnte man noch um zahlreiche Erkrankungen ergänzen. Aber was davon ist wirklich wahr? Und jetzt mal Hand aufs Herz: Wer hatte schon mal einen Patienten, bei dem der Vitamin-D3-Spiegel im Normbereich lag? Vielleicht etwas schwarz-weiß gemalt, aber offensichtlich ist, dass die wissenschaftliche Publikationsplattform Pubmed übervoll von Studien zu Vitamin D3 ist, jedoch endet fast jeder Abstract wie folgt: «Die Empfehlung zu Supplementation zum Schutz vor der Erkrankung X kann auf Grund dieser Daten nicht gegeben werden». Bei Säuglingen ist die Vitamin-D-Gabe Normalität. Umso mehr schreckte uns die Publikation aus dem Jahr 2004 [5] auf, die den epidemiologischen Beweis zu geben schien, dass eine Vitamin-D-Gabe im Säuglingsalter das Allergierisiko erhöht. Darin zeigte sich an einer Geburtskohorte aus Finnland, dass eine Vitamin D-Supplementierung im Kleinkindesalter mit höherer Prävalenz der Sensibilisierung und allergischen Rhinitis im Erwachsenenalter in Verbindung einhergeht.

\section{Vitamin D und das Immunsystem}

Vitamin D3 ist ein Secosteroid mit klassischer Wirkung auf das Skelett und den Mineralienhaushalt. Die aktive Form Calcitriol $\left(1,25(\mathrm{OH})_{2} \mathrm{D}\right)$ entsteht aus der Speicherform Calcidiol (25-OHD). $25-$ $\mathrm{OHD}>50 \mathrm{nmol} / \mathrm{l}$ sind laut Institute of Medicine (IOM) für die Allgemeinbevölkerung ausreichend, um skeletale Mangelerscheinungen zu vermeiden [6].

Vitamin D nimmt auf viele Zellen des Immunsystems Einfluss [7-12]. Die Effekte sind ambigue: Zwar wird eine Immuntoleranz gefördert [8-10], jedoch wird die Proliferation T-regulierender Zellen gehemmt [11] und eine TH2-gewichtete Imbalance induziert [7]; letztere ist mit lgE-vermittelten Allergien assoziiert [13].

\section{Studienergebnisse}

In der Publikation von Savilahti et al. wurde die Assoziation zwischen 25-OHD-Serumkonzentrationen und Allergien im Kindesalter untersucht. Diese neue Studie zeigt, dass die Serum-25-OHDKonzentration bei Geburt und im frühen Kindesalter mit dem Auftreten von IgE-Sensibilisierung und Allergien nichtlinear assoziiert ist: 25-OHD-Konzentrationen des 2. Terzils bei Geburt (21,5-29,5 nmol/I) sind mit einem höheren Risiko für Typ-I-Sensibilisierung unter 2 Jahren und allergischen Erkrankungen bis zum 5. Lebensjahr assoziiert. Das 3. Quartil der 2-Jährigen (51.7-62.6 nmol/l) geht einher mit einem höheren Risiko für Typ-I-Sensibilisierung gegenüber Umweltallergenen und IgE-vermittelten Allergien bis 5 Jahre.

\section{Fazit für die Praxis}

Ein Verzicht der Supplementation von Vitamin D zum Schutz vor Allergien kann aufgrund dieser Daten nicht empfohlen werden. Eine gerade publizierte Metaanalyse zum Thema Vitamin D und Allergien [14] zeigt, dass es wohl Effekte gibt, die allerdings altersund geschlechtsspezifisch sind. Was jedoch für alle Studien gilt, ist: es sind Assoziationen und keine Kausalitäten!

\section{Disclosure Statement}

Hiermit erkläre ich, dass keine Interessenskonflikte in Bezug auf den vorliegenden Kommentar bestehen.

\section{Literatur}

1 Zuk A, Fitzpatrick T, Rosella LC: Effect of vitamin d3 supplementation on inflammatory markers and glycemic measures among overweight or obese adults: A systematic review of randomized controlled trials. PLoS One 2016;11:e0154215.

2 Okereke OI, Singh A: The role of vitamin D in the prevention of late-life depression. J Affect Disord 2016;198:1-14.

3 Aggarwal A, Kallay E: Cross talk between the calcium-sensing receptor and the vitamin d system in prevention of cancer. Front Physiol 2016;7:451.

4 Lips P, Eekhoff M, van Schoor N, et al.: Vitamin D and type 2 diabetes. J Steroid Biochem Mol Biol 2016;S0960-0760(16)30338-7.

5 Hyppönen E, Sovio U, Wjst M, et al.: Infant vitamin d supplementation and allergic conditions in adulthood: northern Finland birth cohort 1966. Ann NY Acad Sci 2004;1037:84-95.

6 Ross AC, Manson JE, Abrams SA, et al.: The 2011 report on dietary reference intakes for calcium and vitamin D from the Institute of Medicine: what clinicians need to know. J Clin Endocrinol Metab 2011;96:53-58.

7 Boonstra A, Barrat FJ, Crain C, et al.: 1alpha,25-Dihydroxyvitamin d3 has a direct effect on naive CD4(+) T cells to enhance the development of Th2 cells. J Immunol 2001;167:4974-4980.

8 Griffin MD, Lutz W, Phan VA, et al.: Dendritic cell modulation by 1alpha,25 dihydroxyvitamin D3 and its analogs: a vitamin D receptor-dependent pathway that promotes a persistent state of immaturity in vitro and in vivo. Proc Natl Acad Sci USA 2001;98:6800-6805.

9 Yip KH, Kolesnikoff N, Yu C, et al.: Mechanisms of vitamin $D_{3}$ metabolite repression of IgE-dependent mast cell activation. J Allergy Clin Immunol 2014;133:1356-1364, 1364.e1-14.

10 Zhang Y, Leung DY, Goleva E: Anti-inflammatory and corticosteroidenhancing actions of vitamin $D$ in monocytes of patients with steroidresistant and those with steroid-sensitive asthma. J Allergy Clin Immunol 2014;133:1744-1752.e1.

11 Khoo AL, Joosten I, Michels M, et al.: 1,25-Dihydroxyvitamin D3 inhibits proliferation but not the suppressive function of regulatory $T$ cells in the absence of antigen-presenting cells. Immunology 2011;134:459-468.

12 Mirzakhani H, Al-Garawi A, Weiss ST, Litonjua AA: Vitamin D and the development of allergic disease: how important is it? Clin Exp Allergy 2015;45: 114-125.

13 Berger A:Th1 and Th2 responses: what are they? BMJ, 2000;321:424.

14 Aryan Z, Rezaei N, Camargo CA Jr.: Vitamin D status, aeroallergen sensitization, and allergic rhinitis: A systematic review and meta-analysis. Int Rev Immunol 2017:36:41-53.

Kontaktadresse: Univ.-Prof. Dr. Claudia Traidl-Hoffmann, Lehrstuhl und Institut für Umweltmedizin, UNIKA-T, Technische Universität München und Helmholtzzentrum München, Neusässer Straße 47, 86156 Augsburg, Deutschland, claudia.traidl-hoffmann@tum.de 